Please do not remove this page

RMIT

UNIVERSITY

\title{
Microdosing psychedelics: Subjective benefits and challenges, substance testing behavior, and the relevance of intention
}

Petranker, Rotem; Anderson, Thomas; Maier, Larissa; Barratt, Monica; Ferris, Jason; Winstock, Adam https://researchrepository.rmit.edu.au/esploro/outputs/9922068506501341/filesAndLinks?institution=61RMIT_INST\&index=null

Petranker, R., Anderson, T., Maier, L., Barratt, M., Ferris, J., \& Winstock, A. (2022). Microdosing psychedelics: Subjective benefits and challenges, substance testing behavior, and the relevance of intention. Journal of Psychopharmacology, 36(1), 85-96. https://doi.org/10.1177/0269881120953994 Document Version: Accepted Manuscript

Published Version: https://doi.org/10.1177/0269881120953994

Repository homepage: https://researchrepository.rmit.edu.au

(C) The Author(s) 2020.

Downloaded On 2023/04/26 20:29:39 +1000 
Microdosing Psychedelics: Subjective Benefits and Challenges, Substance Testing Behavior, and the Relevance of Intention

Petranker, R. ${ }^{1,2}$, Anderson, T. ${ }^{2,3}$, Maier, L. J. ${ }^{4,5}$, Barratt, M. J. ${ }^{6,7}$, Ferris, J. A. ${ }^{8}$, \& Winstock, A. R. ${ }^{9,10}$

${ }^{1}$ Clinical Psychology, York University, Toronto, ON, Canada

${ }^{2}$ Psychedelic Studies Research Program, University of Toronto Mississauga, Mississauga, ON, Canada

${ }^{3}$ Department of Psychology, University of Toronto, Toronto, ON, Canada.

${ }^{4}$ Department of Psychiatry and Weill Institute for Neurosciences, University of California, San

Francisco, CA, United States

${ }^{5}$ Early Postdoc Mobility Grantee (P2ZHP1_17812), Swiss National Science Foundation, Bern,

Switzerland

${ }^{6}$ Social and Global Studies Centre, RMIT University, Australia

7 National Drug and Alcohol Research Centre, UNSW Sydney, Australia

${ }^{8}$ Centre for Health Services Research, Faculty of Medicine, The University of Queensland, Brisbane, Australia

${ }^{9}$ University College London, Gower St, Bloomsbury, London, UK

${ }^{10}$ Global Drug Survey Ltd, London, UK

Please note that both first authors contributed equally to this manuscript.

\section{*Corresponding Author}

Rotem Petranker

Toronto, ON, Canada

Email: rotem@boredomlab.org 


\begin{abstract}
Background: Microdosing psychedelics is the practice of taking small, sub-hallucinogenic doses of LSD or psilocybin-containing mushrooms. Despite its surging popularity, little is known about the specific intentions to start microdosing and the effects of this practice.

Aims: First, we aimed to replicate previous findings regarding the subjective benefits and challenges reported for microdosing. Second, we assessed whether people who microdose test their substances before consumption. Third, we examined whether having an approach-intention to microdosing was predictive of more reported benefits.
\end{abstract}

Methods: The Global Drug Survey (GDS) runs the world's largest online drug survey. Participants who reported last year use of LSD or psilocybin in GDS2019 were offered the opportunity to answer a sub-section on microdosing.

Results: Data from 6,753 people who reported microdosing at least once in the last 12 months were used for analyses. Our results suggest a partial replication of previously reported benefits and challenges among the present sample often reporting enhanced mood, creativity, focus, and sociability. Counter to our prediction, the most common challenge participants associated with microdosing was "None". As predicted, most participants reported not testing their substances. Counter to our hypothesis, approach-intention - microdosing in order to approach a desired goal - predicted less rather than more benefits. We discuss alternate frameworks that may better capture the reasons people microdose.

Conclusion: Our results suggest that the perceived benefits associated with microdosing greatly outweigh the challenges. Microdosing may have utility for a variety of uses while having minimal sideeffects. Double-blind, placebo-controlled experiments are required to substantiate these reports.

Declaration of funding: This research received no specific grant from any funding agency in the public, commercial, or not-for-profit sectors. 


\section{Introduction}

Recent years have seen a groundswell of interest in psychedelics (Pollan, 2018; Waldman, 2017). Evidence is mounting that large doses of substances such as lysergic acid diethylamide (LSD) and psilocybin-containing "magic" mushrooms can help treat depression (Ross et al., 2016), anxiety (Grob et al., 2011), and substance use (Johnson et al., 2014). The popularity of microdosing - consuming small, sub-hallucinogenic amounts of psychedelics such as LSD and psilocybin - has also been rising in recent years, with online forums such as reddit/r/microdosing growing exponentially (Anderson, Petranker, Rosenbaum, et al., 2019). Microdosing has been anecdotally proposed as an alternative to antidepressants (Weiss, 2019) as well as a performance-enhancing supplement (Plante, 2017). Despite the growing public interest in this phenomenon, however, research has been slow to follow this cultural trend with only a few studies published, all in the last three years.

Evidence suggests that microdosing may enhance performance in certain domains, with no serious side-effects reported to date. One study, conducted at a popular microdosing conference, found that administering small amounts (between .22 and $.44 \mathrm{~g}$ ) of psilocybin-containing truffles acutely improved divergent, but not convergent creativity (Prochazkova et al., 2018). An experimental study using small amounts (1-26 $\mu \mathrm{g})$ of LSD found that a single microdose enhanced feelings of "vigor" and social relatedness (Bershad et al., 2019). Neither of these intervention studies reported any adverse effects, consistent with the relatively benign safety profile of these substances (Johnson et al., 2018; Nutt et al., 2010).

Survey work with large sample sizes has been informative regarding the experiences of people who reported microdosing with LSD or psilocybin, and the research potential associated with this practice. One survey reported that people who reported microdosing with LSD or psilocybin score lower in negative emotionality and dysfunctional attitudes and higher on wisdom, open-mindedness, and

creativity (Anderson et al., 2019). Another survey reported somewhat conflicting results, with people who 
reported microdosing with LSD or psilocybin experiencing an increase in negative emotionality, but also reduced depression and stress (Polito \& Stevenson, 2019). Finally, Anderson et al. (2019) created a taxonomy of the most commonly reported benefits and challenges associated with microdosing. They found that the three most commonly reported benefits were improved mood, improved focus, and increased creativity; the three most commonly reported challenges were concerns about illegality, physiological discomfort, and impaired focus (Anderson et al., 2019). We aimed to replicate these findings using a large international sample of people who reported microdosing in the last year.

Due to the concerns around illegality and the many unknowns surrounding microdosing, we were also interested in whether those who report microdosing test their substances. While people who use drugs recreationally are concerned about the content and purity of the substances they use, most of them do not test their substances prior to use. For example, Day et al. (2018) found that 52.8\% (95\% CI [48.3, 57.4]) of people who use illicit drugs at an Australian music festival were concerned about the content and purity of their substances, but only $14.3 \%(95 \%$ CI $[11.1,17.5])$ always test their substances and $37.2 \%(95 \%$ CI $[32.8,41.6])$ never test their substances. Similarly, a recent study found that only $22.7 \%$ $(95 \%$ CI $[19.9,25.5])$ of Australians who use hallucinogens/psychostimulants in nightclubs/festivals and responded to a substance-testing questionnaire reported using testing kits on their substances (Barratt, Bruno, Ezard, \& Ritter, 2018). Accordingly, we hypothesized that most people in our sample would not have tested their substances.

Finally, given the lack of research, we were also interested in participant intention when microdosing. The relevance of intention when taking psychedelics was recognized by Timothy Leary, who popularized the concept of "set and setting" (Pollan, 2018). Intentionality was introduced as one of the main tenets of psychedelic psychotherapy in the first wave of research in the 1960s (Dimascio \& Klerman, 1960; Lasagna 1963, as cited in Hartogsohn, 2016) and remains central in current research (Carhart-Harris et al., 2018). No empirical work has investigated whether there is a relationship between intention and the reported benefits of microdosing. Some theories suggest that for full-dose psychedelic 
experiences, approaching the experience with the intention of engaging rather than circumventing or avoiding the experience improves the outcome of psychedelic experiences (Fadiman, 2011; Zendo, 2017). These theories echo Reinforcement Sensitivity Theory (RST), which includes the Behavioral Approach System (BAS) and the Behavioral Inhibition System (BIS; Gray, 1982). According to RST, some individuals are characterized by sensitivity to rewarding stimuli (high BAS) while others are sensitive to aversive stimuli (high BIS). Those sensitive to reward (BAS) tend to approach risky situations with less concern for potential losses, and those sensitive to aversive stimuli (BIS) are likely to avoid risky situations. In the context of psychedelics use, leaning into a difficult substance-induced experience at the cost of short-term unpleasantness has been suggested as a predictor of long-term positive outcomes (Fadiman, 2011). We subsequently hypothesized that an approach-motivation would more positive experiences for people who microdose. While we were unable to directly measure approach/avoidance

motivation in the present study, we measured initial intention to microdose, indexing these intentions with an a priori approach vs avoid motivation dichotomy.

\section{Hypotheses}

The present study had three main aims. First, we aimed to validate our previous findings on perceived benefits and challenges of microdosing with LSD and psilocybin (Anderson, Petranker, Christopher, et al., 2019) in a larger, international sample (H1 \& H2). Second, we wanted to assess whether people who microdose test substances purchased through the black market prior to use (H3). Third, this study examined whether specific intentions towards microdosing is predictive of deriving more benefit from this practice; specifically, that an approach-motivation would be related to more subjective microdosing-related benefits than an avoidance-motivation (H4). 
H1: The three most common benefits of microdosing will be improved mood, improved focus, and increased creativity.

H2: The three most common challenges of microdosing will be concerns about illegality, physiological discomfort, and impaired focus.

H3: The majority of participants who microdose will not have tested the substance they used to microdose.

H4: Participants who report an approach-intention will report significantly more benefits than participants who report an avoidance-intention.

\section{Methods}

\section{Pre-Registration}

Prior to data collection, the methods and hypotheses reported here were pre-registered on the Open Science Framework (OSF; https://osf.io/vp4nw/). No hypotheses were registered regarding the relative benefits of microdoses versus higher doses, so results of that question are reported as descriptive, not inferential. Likewise, predictions regarding anxiety diagnoses were not pre-registered and should be considered exploratory. The data reported here concerns respondents from the 2019 Global Drug Survey who reported microdosing in the last 12 months.

\section{Study design and recruitment}

The Global Drug Survey (GDS) is the world's largest anonymous online drug survey, which has been conducted annually since 2012 (Barratt et al., 2017). GDS2019 was available in 19 languages (English, Albanian, Azerbaijani, Brazil, Czech, Danish, Dutch, Finnish, French, German, Hungarian, Italian, Lithuanian, Portuguese, Romanian, Serbian, Slovak, Spanish, and Turkish) and was promoted through partnerships with media partners, harm reduction organizations, and via social media. As a self- 
nominating sample, our population represents a non-probability sample and should not be used to infer the prevalence of drug use and related activities to the general public. Ethics approval was obtained ${ }^{1}$.

\section{Measures}

The GDS starts with demographics questions, followed by an extensive drug screen (100+ drugs) where participants indicate when they last used specific drugs (never, more than 12 months ago, within the last 12 months but not last month, or within the last 30 days). Participants who indicated the use of either LSD or psilocybin-containing mushrooms during the last 12 months were then offered the opportunity to complete an in-depth section including questions specifically about their experience of microdosing with either substance using a previously compiled list of possible benefits and challenges (Anderson et al. 2019). Microdosing was defined as less than 20 micrograms of LSD and less than 0.2 grams of Magic Mushrooms (MM).

\section{Microdosing Benefits}

The benefits and challenges questions used in this survey were based on our empirically derived taxonomy (Anderson et al., 2019, see Table 1). We asked participants to indicate which benefits from the following list applied to their experience of microdosing (missing values were excluded from the analysis): (1) Enhanced mood, reduced depression symptoms; (2) Enhanced focus; (3) Enhanced creativity and/or curiosity; (4) Enhanced productivity, motivation, or confidence; (5) Enhanced energy and/or alertness; (6) Enhanced empathy, sociability or communication skills; (7) Enhanced mental clarity

\footnotetext{
${ }^{1}$ Ethical approval for GDS2019 was obtained from the University College London (11671/001), University of Queensland (No: 2017001452) and from the University of New South Wales (HREC HC17769) Research Ethics Committees. GDS surveys are labelled by the year that the data was analyzed and released. Responses were collected for ten weeks between October 29, 2018 and January 10, 2019.
} 
and/or memory; (8) Enhanced sight, smell, hearing, athletic performance or sleep; (9) Reduced stress; (10) Reduced anxiety, including social anxiety; (11) Reduced substance dependence symptoms; (12)

None; I experienced no effect at all or negative effect.

Table 1

GDS 2019 Benefits and Challenges questions based on empirical taxonomy from Anderson et al. (2019).

\begin{tabular}{|c|c|c|}
\hline Category & Anderson et al (2019) & GDS2019 Response Options \\
\hline Creativity & Creativity & Enhanced creativity and/or curiosity \\
\hline Illegality & Illegality & Legal consequences \\
\hline Mood & Improved Mood & Enhanced mood, reduced depression symptoms \\
\hline Mood & Impaired Mood & Negative mood, irritability or instability \\
\hline Self & Self-Efficacy & Enhanced productivity, motivation, or confidence \\
\hline Self & Self-Interference & Dissociation and/or rumination \\
\hline Focus & Improved Focus & Enhanced focus \\
\hline Focus & Impaired Focus & Reduced Focus \\
\hline Social & Social Benefits & Enhanced empathy, sociability or communication skills \\
\hline Social & Social Interference & Social Problems \\
\hline Energy & Improved Energy & Enhanced energy and/or alertness \\
\hline Energy & Impaired Energy & Restlessness and/or fatigue \\
\hline Cognitive & Cognitive Benefits & Enhanced mental clarity and/or memory \\
\hline Cognitive & Cognitive Interference & Mental confusion, memory problems, or racing thoughts \\
\hline
\end{tabular}




\begin{tabular}{lll}
\hline Anxiety & Reduced Anxiety & Reduced anxiety, including social anxiety \\
Anxiety & Increased Anxiety & Increased anxiety, including social anxiety \\
Symptoms & Reduced Symptoms & Reduced substance dependence symptoms \\
Symptoms & Increased Symptoms & Substance dependence symptoms and hard comedown \\
Other & Other Benefits & Reduced stress \\
Other & Other Challenges & Unpredictable effects and/or negative drug interactions \\
Physiological & Physiological & Enhanced sight, smell, hearing, athletic performance or sleep \\
& Enhancement & \\
Physiological & Physiological Discomfort & Stomach pain, headache, sleep problems, loss of appetite \\
None & None (Benefit) & None; I experienced no effect at all or negative effect \\
& None (Challenge) & None, I experienced no side effects \\
\hline
\end{tabular}

\section{Microdosing Challenges}

We asked participants to indicate which challenges from the following list (see Table 1) applied to their experience of microdosing (missing values were excluded from the analysis): (1) Negative mood, irritability or instability; (2) Reduced focus; (3) Legal consequences; (4) Restlessness and/or fatigue; (5) Social problems; (6) Mental confusion, memory problems, or racing thoughts; (7) Stomach pain, headache, sleep problems, loss of appetite; (8) Dissociation and/or rumination; (9) Increased anxiety, including social anxiety; (10) Substance dependence symptoms and hard comedown; (11) Unpredictable effects and/or negative drug interactions (12) None; I experienced no side effects. 


\section{Substance Testing Behavior}

Participants were asked "Did you usually analyze the substance used to microdose with?" and could use the following 5 responses: 1) "Yes, and it was the substance I expected to use", 2) "Yes, but it was a different substance than I expected, but I still microdosed", 3) "No, but I tried a full-dose of the same batch beforehand and it seemed to be the substance I expected", 4) "No, but I bought from a source I consider reliable", 5) "No, and I cannot be sure". Responses 1-2 were treated as having tested substances and responses 3-5 were treated as not having tested substances.

\section{The Relevance of Intention to Reported Benefits}

To measure initial intention, participants were asked to pick one of the following reasons for initiation of microdosing: (1) to enhance creativity and/or ingenuity; (2) to improve mood and/or overall life satisfaction; (3) to avoid boredom; (4) to escape negative feelings, e.g. depression, anxiety; (5) to improve my relationships with myself and/or others in my life; (6) to get away from bad habits and unhealthy behaviours; (7) to be more productive and effective; (8) to treat ADHD symptoms; (9) just curious; (10) to stop problematic substance use; (11) I don't know / I didn't have a particular reason.

We indexed intentions as either approach or avoid (see results section, Table 4). Responses 1, 2, 5, 7, and 9 were considered approach intentions and responses $3,4,6,8$, and 10 were considered avoid intentions; option 11 was not coded in either direction. Benefits were calculated as a sum of benefits reported in the "benefits and challenges" section. Some respondents selected benefits, then also selected "None; I experienced no effect at all or negative effect". This was not foreseen in the pre-registration and occurred infrequently (LSD: $\mathrm{N}=26 ; \mathrm{MM}: \mathrm{N}=13$ ). In these cases, the number of benefits was set to zero to favour a conservative estimate. 


\section{The Relative Benefits of Microdoses versus Higher doses}

Participants were asked "How would you compare the beneficial effects of microdosing with a full dose of [magic mushrooms (psilocybin) | LSD]?" and could use the following 6 responses: 1) "Microdosing is much more beneficial for me", 2) "Microdosing is somewhat more beneficial for me", 3) "A full dose is somewhat more beneficial for me", 4) "A full dose is much more beneficial for me", 5) "Both are the same for me", and 6) "Neither is beneficial for me".

\section{Microdosing and anxiety disorder.}

Participants were asked "Have you ever been diagnosed with a mental illness?" and could indicate a diagnosis of "Anxiety".

\section{Results}

\section{Sample characteristics}

Unique responses from 123,814 participants in 215 countries and territories were recorded in the Global Drug Survey (GDS) 2019. Most participants were from Germany $(\mathrm{N}=35,038)$, the USA ( $=$ 11,247), New Zealand $(\mathrm{N}=9,997)$, Australia $(\mathrm{N}=7,864)$, Denmark $(\mathrm{N}=7,738)$, or England $(\mathrm{N}=6,586)$. Out of this larger sample, 4,783 reported having microdosed LSD in the last 12 months ( $74 \%$ male; median age $=23, \mathrm{SD}=8.43), 2,832$ participants report having microdosed psilocybin-containing "magic" mushrooms $(\mathrm{MM})$ in the last 12 months $(71 \%$ male; median age $=26, \mathrm{SD}=9.66)$, and 862 participants reported microdosing both in the last 12 months $(73 \%$ male; median age $=26, \mathrm{SD}=9.53)$. Microdosing was defined as less than 20 micrograms of LSD and less than 0.2 grams of MM. Most people who reported microdosing were from the USA (LSD: $N=997, M M: N=732$ ), Germany (LSD: $N=843$, MM: $\mathrm{N}=397$ ), or Australia (LSD: $\mathrm{N}=357, \mathrm{MM}: \mathrm{N}=200$ ) and there were over 100 participants who reported microdosing LSD from England, Brazil, Finland, New Zealand, Switzerland, Canada, Colombia, Norway, and France and over 100 participants who reported microdosing MM from Canada, Finland, and England. 


\section{Most Commonly Reported Benefits and Challenges}

\section{Microdosing Benefits.}

In partial support of H1, the two most commonly reported benefits of microdosing with LSD and MM were improved mood (LSD: 51.23\%, 95\% CI: [49.81\%, 52.64\%]; MM: 56.84\%, 95\% CI: [55\%, 58.66\%]) and creativity (LSD: 46.61\%, 95\% CI: [45.2\%, 48.03\%]; MM: 49.29\%, 95\% CI: [47.45\%, 51.14\%]). Counter to H1, the third most commonly reported benefit for LSD was increased energy (44.54\%, 95\% CI [43.13\%, 45.95\%], and the third most commonly reported benefit for MM was social benefits $(41.03 \%, 95 \% \mathrm{CI}:[39.23 \%, 42.86 \%])$. Counter to H1, improved focus was not in the top three; it was the $6^{\text {th }}$ most commonly reported benefit among participants who microdosed with LSD $(39.33 \%, 95 \%$ CI: $[31.41 \%, 34.08 \%])$, and the $9^{\text {th }}$ most commonly reported benefit for the MM group $(30.01 \%, 95 \%$ CI: $[28.35 \%, 31.73 \%])$.

See Table 2 for reported benefits of microdosing with LSD and MM:

Table 2

Reported microdosing benefits by substance: Count and Percent (\%)

\begin{tabular}{lcc}
\hline Benefit & LSD (\%) & MM (\%) \\
\hline Enhanced mood, reduced depression symptoms & $2443(51.23)$ & $1604(56.84)$ \\
Enhanced creativity \& curiosity & $2223(46.61)$ & $1391(49.29)$ \\
Enhanced energy \& alertness & $2124(44.54)$ & $1110(39.33)$ \\
Enhanced empathy, sociability or communication skills & $1797(37.68)$ & $1158(41.03)$ \\
Enhanced productivity, motivation, or confidence & $1706(35.77)$ & $955(33.84)$ \\
Enhanced focus & $1561(32.73)$ & $847(30.01)$ \\
Reduced stress & $1475(30.93)$ & $1079(38.24)$
\end{tabular}


Reduced (social) anxiety

Enhanced mental clarity \& memory

Enhanced sight, smell, hearing, athletic performance, or sleep

Reduced substance dependence symptoms

None, I experienced no effect at all or negative effect
$1443(30.26)$

$1374(44.89)$

$1260(26.42)$

$566(11.66)$

194 (4.07)

4769
$937(33.20)$

$825(29.23)$

$879(31.15)$

$414(14.67)$

$153(5.42)$

Total number of responses

2822

Note. Participants were able to select more than one response option. 


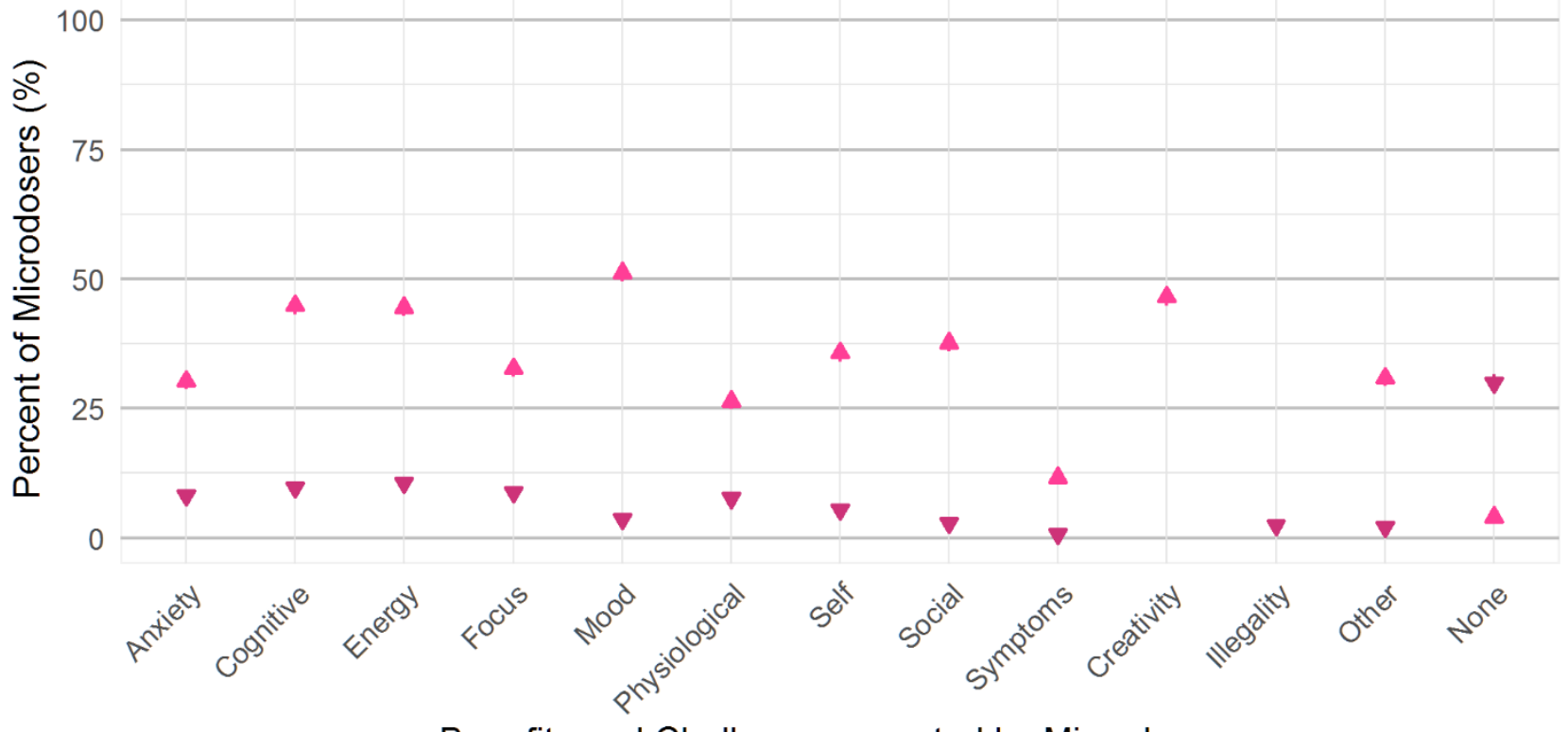

Benefits and Challenges reported by Microdosers

\section{LSD Benefits $\neg$ LSD Challenges}

Figure 1. Percent of participants who microdosed LSD reporting benefits and challenges of microdosing by category $(\mathrm{N}=4,769)$. Note that participants could select more than one benefit or challenge thus percentages add to over $100 \%$. Error bars indicate $95 \%$ CI around population estimate and are typically subsumed by the point due to the large sample size.

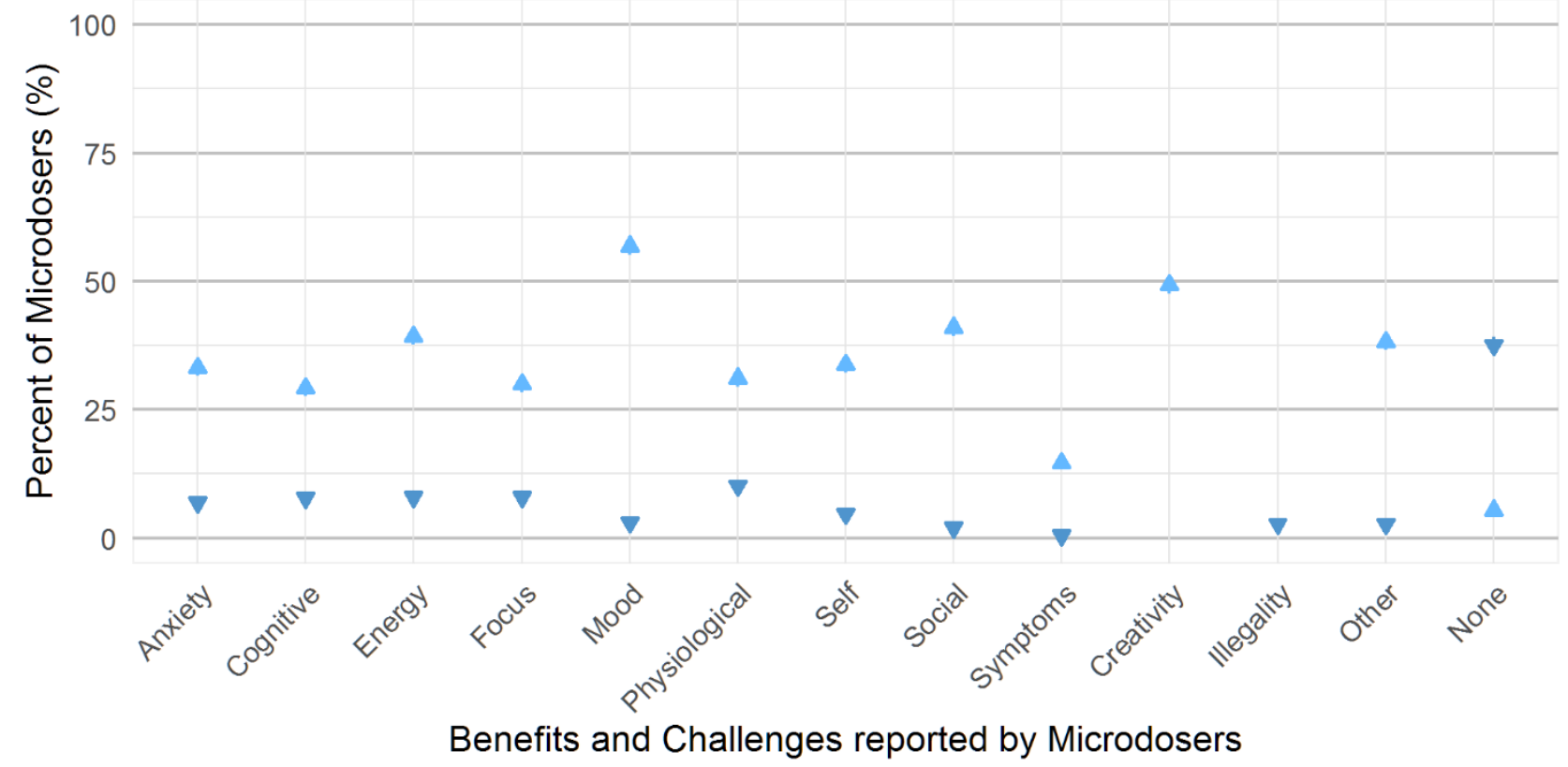

MM Benefits $\neg$ MM Challenges

Figure 2. Percent of participants who microdosed MM reporting benefits and challenges of microdosing by category $(\mathrm{N}=2,822)$. Note that participants could select more than one benefit or challenge thus percentages add to over $100 \%$. Error bars indicate $95 \%$ CI around population estimate and are typically subsumed by the point due to the large sample size. 


\section{Microdosing Challenges}

Counter to $\mathrm{H} 2$, the most commonly reported challenge for both substances was not illegality: it was "None, I experienced no side effects" (for LSD, 29.92\%, 95\% CI: [28.64, 31.24]; for MM: 37.53\%, 95\% CI: $[35.76 \%, 39.33 \%])$. We subsequently detail the next most commonly reported challenges after "none" below.

For LSD, the next three most commonly reported challenges were restlessness or fatigue (impaired energy: $10.59 \%, 95 \% \mathrm{CI}:[9.75,11.49])$, mental confusion, memory problems, or racing thoughts (cognitive interference: 9.69\%, 95\% CI: [8.88, 10.56]), and impaired focus $(8.87 \%, 95 \%$ CI: $[8.10,9.71]$ ), which does not support H2. For MM, the next three most commonly reported challenges were physiological discomfort (10.21\%, 95\% CI: [9.14\%, 11.38\%]), impaired focus $(7.97 \%, 95 \% \mathrm{CI}$ : $[7.03 \%, 9.03 \%])$, and impaired energy $(7.97 \%, 99 \% \mathrm{CI}:[7.03 \%, 9.03 \%])$, the first two of which partially support $\mathrm{H} 2$.

Counter to $\mathrm{H} 2$, illegality was not in the top three; it was the $10^{\text {th }}$ most commonly reported challenge in the LSD group $(2.43 \%, 95 \%$ CI $[2.03 \%, 2.91 \%])$, and the $9^{\text {th }}$ most commonly reported challenge for the MM group (1.64\%, 95\% CI [1.31\%, 2.04\%]. In the LSD group, physiological discomfort was the $6^{\text {th }}$ most commonly reported challenge. See Table 3 for reported challenges for LSD and MM.

Table 3

Reported microdosing challenges by substance: count and percent (\%)

\begin{tabular}{lll}
\hline Challenge & LSD (\%) & MM (\%)
\end{tabular}

None, I experienced no side effects

$1427(29.92) \quad 1059(37.53)$

Restlessness and/or fatigue

$505(10.59)$

$225(7.97)$

Mental confusion, memory problems, or racing thoughts

$462(9.69)$ 
Reduced Focus

$423(8.87)$

$225(7.97)$

Increased anxiety, including social anxiety

$389(8.16)$

$195(6.91)$

Stomach pain, headache, sleep problems, loss of appetite

$366(7.67)$

$288(10.21)$

Dissociation or rumination

$263(5.51)$

$132(4.68)$

Negative mood, irritability or instability

$173(3.63)$

$85(3.01)$

Social Problems

$136(2.85)$

$58(2.06)$

Legal Consequences

$116(2.43)$

$78(2.76)$

Unpredictable effects and/or negative drug interactions

$101(2.12)$

$78(2.76)$

Substance dependence symptoms and hard comedown

$34(0.71)$

$15(0.53)$

Total number of responses

4769

2822

Note. Participants were able to select more than one response option, such that total number of reports amounts to more than the number of participants.

\section{Substance Testing Behaviour}

Supporting H3, less than half of respondents reported testing their substances (LSD: $37.88 \%$, 95\% CI: [35.66\%, 40.14\%], MM: 46.31\%, 95\% CI [44.13\%, 48.51\%]; see Figure 3). Specifically, participants reported "Yes, it was the substance I expected to use" (LSD: 36.87\%, 95\% CI [35.19\%, 38.59\%], MM: 45.30\%, 95\% CI [43.12, 47.5]), "Yes, but it was a different substance than I expected, but I still microdosed" (LSD: 1.00\%, 95\% CI [0.71\%, 1.42\%], MM: 1.01\%, 95\% CI [0.65\%, 1.56\%]), "No, but I tried a full-dose of the same batch beforehand and it seemed to be the substance I expected" (LSD: 14.14\%, 95\% CI[12.96\%, 15.41\%], MM: 11.26\%, 95\% CI: [9.94\%, 12.73\%]), "No, but I bought from a source I consider reliable" (LSD: 35.55\%, 95\% CI [33.88\%, 37.25\%], MM: 31.52\%, 95\% CI [29.51\%, 
33.6\%]), and "No, and I cannot be sure" (LSD: 12.43\%, 95\% CI[11.32\%, 13.64\%], MM: 10.91\%, 95\% CI $[9.61 \%, 12.36 \%])$.

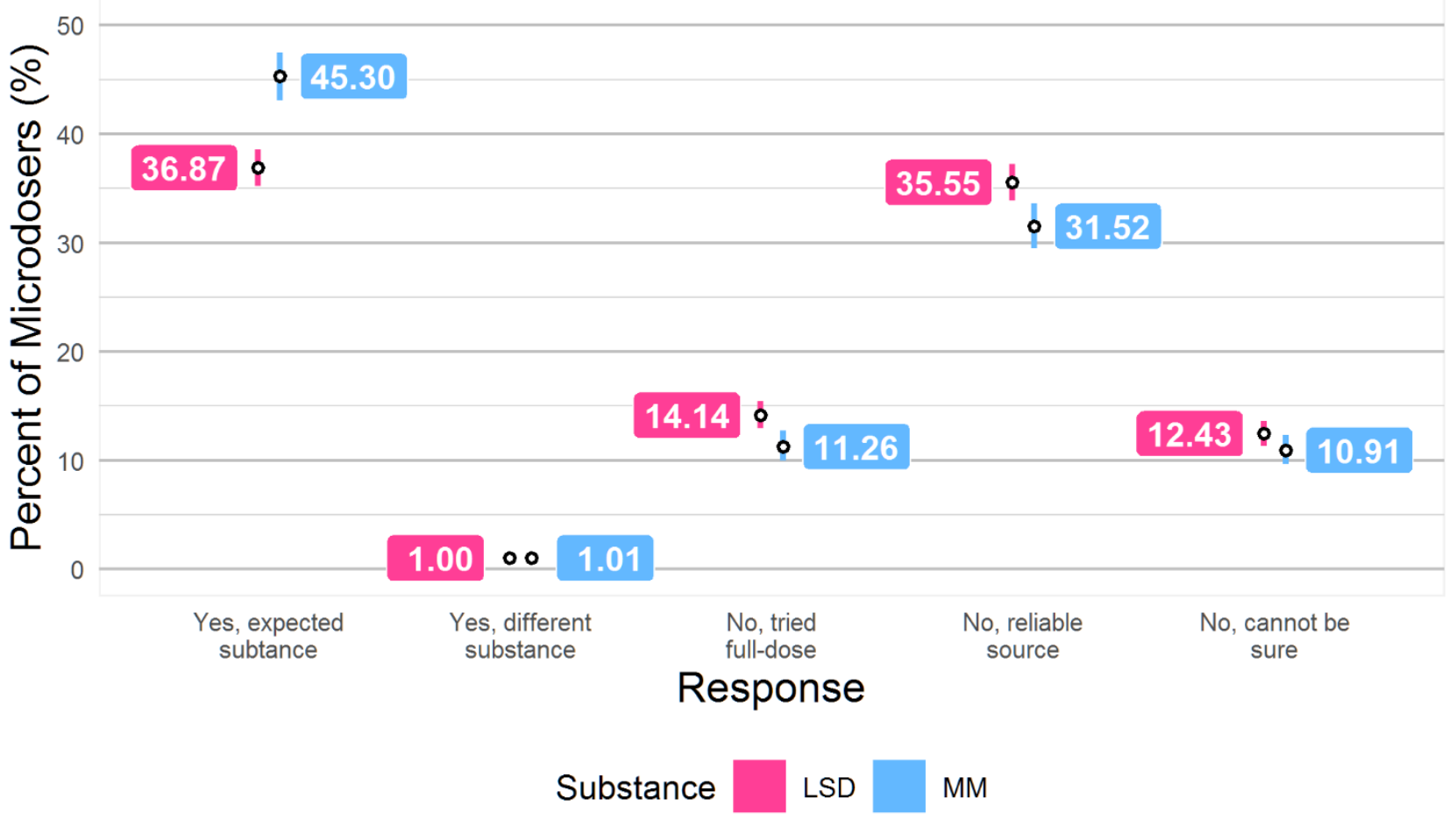

Figure 3. Percent of participants who reported microdosing and their substance testing prior to use. Error bars indicate $95 \%$ CI around population estimate. For full question text, see body.

\section{The Relevance of Intention to Reported Benefits}

Participants had mostly approach-intention for both LSD (80.31\%, 95\% CI: [78.90, 81.64]) and MM (78.82\%, 95\% CI: [77.02, 80.52]); see Table 3 for a breakdown of individual reasons to initiate microdosing. In contrast and against $\mathrm{H} 4$, however, approach-intention was predictive of fewer benefits for both LSD and MM. Using a Wilcoxon Signed-Rank test, approach-intention was predictive of less reported benefits for those who reported microdosing LSD compared to avoid-intention (median approach benefits: 5; media avoid benefits: 6 ; $\mathrm{W}=524081, \mathrm{p}<0.001$; $\mathrm{r}$ 0.07). For those who reported microdosing MM, approach was also predictive of fewer benefits than avoid (median approach benefits: 5; median avoid benefits: $6, \mathrm{~W}=217071, \mathrm{p}<0.001 ; \mathrm{r}: 0.10)$. 
Table 4

Reasons to initiate microdosing by substance

\begin{tabular}{|c|c|c|c|}
\hline Intention & Reason & $\operatorname{LSD}(\%)$ & MM (\%) \\
\hline$\overline{\text { Approach }}$ & To improve mood and/or overall life satisfaction & $821(25.42)$ & $606(28.97)$ \\
\hline Approach & Just curious & $798(24.71)$ & $541(25.86)$ \\
\hline Approach & To enhance creativity and/or ingenuity & $575(18.70)$ & $288(13.77)$ \\
\hline Approach & To be more productive and effective & $268(8.30)$ & $81(3.87)$ \\
\hline Avoid & To escape negative feelings, e.g. depression, anxiety & $196(6.07)$ & $179(8.56)$ \\
\hline N/A & I don't know / I didn't have a particular reason & $179(5.54)$ & $133(6.36)$ \\
\hline Approach & $\begin{array}{l}\text { To improve my relationships with myself and/or } \\
\text { others in my life }\end{array}$ & $132(4.09)$ & $133(6.36)$ \\
\hline Avoid & To treat ADHD symptoms & $92(2.85)$ & $31(1.48)$ \\
\hline Avoid & To avoid boredom & $77(2.38)$ & $40(1.91)$ \\
\hline Avoid & $\begin{array}{l}\text { To get away from bad habits and unhealthy } \\
\text { behaviours }\end{array}$ & $72(2.23)$ & $40(1.91)$ \\
\hline Avoid & To stop problematic substance use & $20(0.62)$ & $20(0.96)$ \\
\hline & Total number of responses & 3230 & 2092 \\
\hline
\end{tabular}

Note. Participants were able to select one option only. 


\section{The Relative Benefits of Microdoses versus Higher doses}

Respondents to this question (LSD N=3085; $\mathrm{MM} \mathrm{N}=1992$ ) reported that full doses provided more benefits than microdosing for both $\operatorname{LSD}(42.3 \%, 95 \% \mathrm{CI}[40.57,44.05])$ and MM $(42.37 \%, 95 \% \mathrm{CI}$ $[40.22,44.55])$. Responses were not one-sided, however, as many participants reported that microdosing was more beneficial (LSD: 30.73\%, 95\% CI [29.13, 32.38]; MM 31.28\%, 95\% CI [29.28, 33.35]) and a substantial proportion reported equivalent benefits regardless of dose (LSD: 23.95\%, 95\% CI [22.48, 25.49]; MM: 22.59\%, 95\% CI [20.81, 24.48]). Response patterns across substances were remarkably similar, within a few percent for each response option (see Figure 4). Very few participants reported that neither dose was beneficial (LSD: 3.01\%, 95\% CI [2.47, 3.68]; MM: 3.77\%, 95\% CI [3.01, 4.69]).

\section{Microdosing and anxiety disorder.}

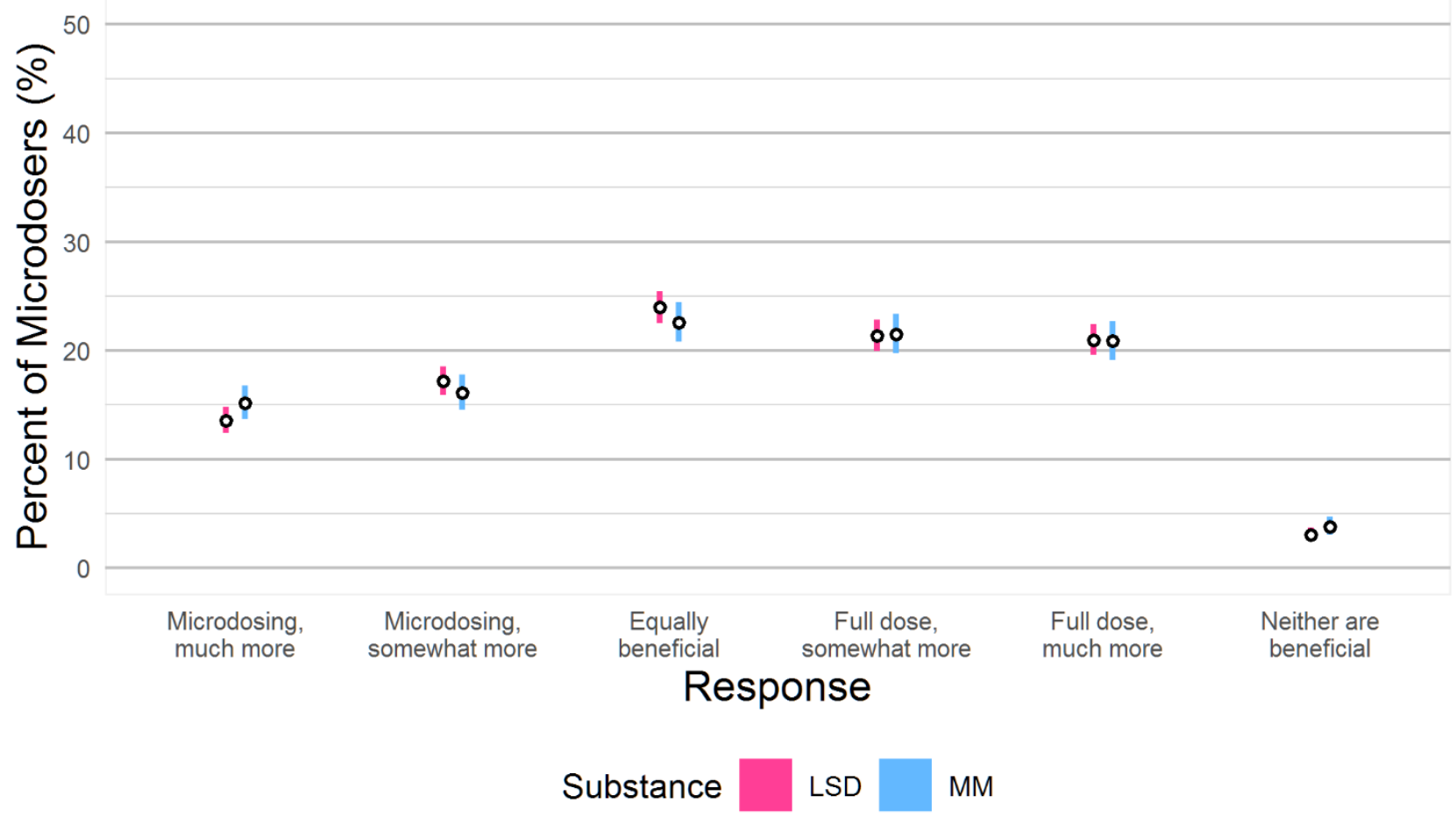

Figure 4. Percent of participants who microdosed reporting dose-related benefits. Error bars indicate $95 \% \mathrm{CI}$ around population estimate. For full question text, see Methods section.

We used a bivariate logistic regression model and odds-ratio testing to determine whether microdoser status was predictive of an anxiety disorder diagnosis. Contrary to our hypothesis, neither 
microdosing LSD $(b=0.03, p=.73 ;$ OR $=1.03,95 \%$ CI: $[0.993,1.06])$ nor MM $(b=-0.007, p=.12 ;$ OR $=0.993,95 \% \mathrm{CI}:[0.958,1.03])$ were predictive of an anxiety disorder diagnosis.

\section{Discussion}

This study had three primary aims: validating previous research on the most common benefits and challenges associated with microdosing with LSD and psilocybin, informing harm reduction initiatives of substance analysis practices for people who microdose, and assessing the utility of approach-avoid intentions when considering the relationship between initial intentions and reported microdosing benefits.

\section{Most Commonly Reported Benefits and Challenges}

\section{Microdosing Benefits}

We expected the three most commonly reported benefits would match the findings of our previous empirical taxonomy, i.e. improved mood, improved focus, and creativity (Anderson et al., 2019). Improved mood and creativity were correctly predicted, but improved focus was considerably less commonly reported than expected. Additional benefits that were commonly reported in the present sample (improved energy, social benefits) were also highly reported in our previous sample $\left(5^{\text {th }}\right.$ and $6^{\text {th }}$ most common respectively). Similar but non-identical findings are not surprising given that the methodologies used in the two studies were quite different; Anderson et al. (2019) used a qualitative grounded theory approach to develop categories from free text responses whereas the present study used closed-ended focal questions based off the categories that were developed. Improved mood and creativity being so commonly reported across both study methodologies speaks to their importance as high-potential research avenues for future microdosing trials.

The benefits reported here converge with the wider literature. Prochazkova et al. (2018) found that participants performed significantly better on a divergent creativity task following a small dose of 
psilocybin. Polito and Stevenson (2019) found that on the day participants reported microdosing, their self-reported creativity, focus, and well-being were significantly elevated compared to baseline. Bershad et al. (2019) found that acute effects of a microdose of LSD included an increased feeling of vigor and friendliness, similar to our findings of increased energy and social benefits. Bershad et al. also found that those who microdosed LSD rated positively-valenced images as less positive. The literature is not of one voice, however, as a recent survey that found some people who report microdosing with psychedelic substances experienced negative psychological effects (Hutten et al., 2019).

\section{Microdosing Challenges}

We expected the three most commonly reported challenges would be concerns about illegality, physiological discomfort, and impaired focus, as we found previously (Anderson et al., 2019). Counter to our predictions, the most commonly reported challenge was "None" and over half of participants experienced no noticeable adverse side-effects from microdosing. That "None" is such a common response here is a promising finding, one that went undetected in the open qualitative reports (Anderson, Petranker, Christopher, et al., 2019). Many respondents left several of the qualitative challenges options blank and we refrained from coding these; it may be that some of those respondents had no challenges to report, as the present sample reported. In the present sample, physiological discomfort and impaired focus were correctly predicted as top three challenges in MM microdosing, but impaired focus was fourth with LSD microdosing and physiological discomfort was sixth. These findings align well with a recent survey that found microdosing is associated with reported negative physiological effects including nausea and dizziness (Hutten et al., 2019).

Illegality was considerably less commonly reported than expected. This was surprising given the wide margin by which it was the most reported challenge in our previous work (Anderson, Petranker, Christopher, et al., 2019). These discrepant findings may be due to limitations in naming conventions for this study or due to an over-general category in the previous work. The original illegality category from 
Anderson et al (2019) conceptually included illegality per se, but also included the consequences thereof, i.e. substance ambiguity due to lack of regulation and the social stigma surrounding substance use. The current operationalization of illegality reduced this category to "Legal consequences", which may have overly limited the scope of the category. It is also possible that the original category was too broad and these sub-categories would be better operationalized separately as "Legal consequences", "Substancerelated issues (e.g. dosing, purity)", and "Social stigma" in future research. Another explanation for this discrepancy is that the current sample was drawn from the GDS, which attracts more drug-involved populations, and such populations may already have dismissed/factored in 'illegality' risks due to their current use of other illegal drugs in addition to microdosing.

The most commonly reported challenges in this sample, alongside physiological discomfort and impaired focus, were impaired energy (restlessness and/or fatigue) and cognitive interference (i.e. mental confusion, memory problems, or racing thoughts). Some of these challenges could plausibly relate to dosing too high when attempting to microdose. The lack of precision dosing available when consuming LSD purchased from the black market or the natural variance in MM potency could contribute to higher doses than intended while remaining sub-hallucinogenic. Psychedelics are central nervous system stimulants that may result in unintentional overstimulation in the form of restless energy or racing thoughts. These differences may also arise from variation in how individuals metabolize psychedelic substances, or even the "set and setting" of the microdose. Future research should endeavour to characterize the ideal dose-range and optimal conditions for microdosing such that people who microdose can maximize benefits and minimize challenges.

\section{Benefits and Challenges - Future Directions}

Our results concur with other studies that suggest the benefits related to microdosing may be considerable, especially compared to the challenges, which appear relatively benign. Convergent findings demonstrate that people who microdose consistently report improved mood and creativity. If it is indeed 
the case that small, sub-hallucinogenic doses of LSD or psilocybin can enhance these domains, current theories proposing that psychedelics must be consumed in large doses to provide beneficial effect (Carhart-Harris \& Friston, 2019) need to be revised.

\section{Substance Testing Behavior}

Our extension of the literature examined whether those who microdose psychedelics test their substances. Our findings indicate that, as predicted, most individuals did not test their substances prior to use. In our sample, fewer than half of participants tested their substances. Participants reported taking approximately $10 \%$ of a recreational dose, which may have reduced the perceived harm potential of consuming unknown substances. Furthermore, our survey did not ask participants how exactly they tested their substances (e.g. using an at-home reagent testing, an on-site festival testing, or by sending a sample away to a lab), so their exact testing procedures remain unknown. Additionally, it is possible that many participants were unaware of substance-testing kits or services and were relying on buying their substance from a source they trusted. Indeed, this was the second most popular reported testing behaviour, in agreement with the finding that this approach is widespread (Davis \& Rosenberg, 2017; Johnston et al., 2006).

This finding is unsurprising considering previous work on similar substances. Johnston et al. (2006) found that one in five people who used 3,4-Methylenedioxymethamphetamine (MDMA) analyzed their substances. Similarly, a large survey that asked participants about pill-testing habits revealed that fewer than half of respondents used anything other than hearsay to validate the purity of the substance they were using (Davis \& Rosenberg, 2017). Using samples of frequent MDMA users, they showed that $74 \%$ of Americans tested their substances using a reagent kit, while only $34 \%$ of UK residents tested theirs. The findings from the field are somewhat inconsistent, suggesting that substance-testing behavior is not necessarily widespread and may differ dramatically between geographical locations. 
In the case of LSD on blotter paper, black market adulterants may include cheaper psychedelics that are active at similarly small doses, such as substituted dimethoxyamphetamines (DOx family) and substituted N-benzyl-phenethylamines (25x-NBOMe family). Unfortunately, these substance do not share the benign safety profile of LSD and have led to hospitalizations and overdoses (Suzuki et al., 2015). Concerning MM, it may be difficult to substitute the whole dried mushroom, though powdered mushrooms could plausibly be adulterated with other psychedelics. One central issue of import faced by people who microdose is dose consistency and ensuring that a large dose is not consumed unintentionally due to the unregulated and untested nature of these substances.

\section{The Relevance of Intention to Reported Benefits}

The results obtained from this sample directly contradict our predicted relationship. Participants who started microdosing to avoid a negatively-appraised state or behavior rather than approach a positively-appraised state or behavior reported significantly more benefits from microdosing. We provide three possible explanations: perhaps our operationalization of summing benefits was inadequate, perhaps the approach-avoid dichotomy is not theoretically applicable to microdosing and different conceptualizations would add clarity, or perhaps these findings really reveal something surprising about microdosing with an avoidance mindset: the importance of being prepared to change.

It is possible that counting the number of benefits is not an effective operationalization of outcomes as benefits may vary on a spectrum of how valuable or important they are to the participants. For example, if a participant credits microdosing with a transformative alleviation of depression symptoms or a desired enhancement in creativity and productivity, whether this participant also reports enhanced physical senses may be clinically and subjectively negligible. This explanation seems plausible as in our previous work, we found that participants who microdosed with MM reported significantly more subjective importance of benefits compared to participants who microdosed with LSD. In the present data, a higher percentage of participants who microdosed LSD endorsed more benefits (see Figures 1 \& 
2), but we did not measure subjective importance of benefits. Unfortunately, our previous work did not assess intention to microdose so cannot offer more insight here (Anderson et al., 2019). More precise measurements and validated scales planned for upcoming clinical trials on microdosing can be expected to be more informative than the present operationalization.

Another possible reason for this pattern of results is that the approach-avoid framework may not be theoretically applicable in this context. Gray's approach-avoid framework has been used as a trait-level construct to measure individual differences related to certain psychopathologies, e.g. high BAS is related to risk-taking behavior, such as substance use (Bijttebier, Beck, Claes, \& Vandereycken, 2009), whereas individuals with high BIS tend toward anxiety disorders (Carver \& White 1994). This theory also dovetails with mainstream psychedelic theories that suggest individual differences and expectancy drive some psychedelic effects (Carhart-Harris et al., 2018). Indeed, Carhart-Harris and colleagues specifically mention that the context in which individuals microdose is yet unstudied and may be an important addition to the literature.

We hypothesized that those intending to approach pleasant states —improved mood and life satisfaction, curiosity, creativity, enhanced productivity, or better relationships - would reap more benefits from their microdosing practice than those trying to avoid aversive experiences — depression, anxiety, or attention deficit hyperactivity disorder (ADHD) symptoms, boredom, bad habits, unhealthy behaviors, or problematic substance use - but our results contradict this prediction. Instead, the data suggest that avoid-intentions were predictive of more benefits. It is possible that conceptualizing intention along approach-avoid dimensions is less applicable than initially thought. Indeed, other dimensions of intention, such as those of self-improvement and self-medication, may provide more fruitful interpretations (Petranker et al., 2020). It may be that participants with self-improvement intentions could face ceiling effects or diminishing returns if they are already doing well in life. Conversely, participants faring particularly poorly when starting to microdose would benefit from regression to the mean or from alleviating downstream issues by addressing primary causes. For example, ADHD and boredom are 
related to other mental health concerns (Eastwood et al., 2012). Someone using microdosing to selfmedicate their ADHD symptoms could report an improvement not only in these symptoms, but also various comorbid issues or downstream issues caused by their disorder, whether or not it was their intention to treat these issues. In other words, it is plausible that intending to self-medicate depression, anxiety, or problematic substance use, all of which are often comorbid with each other and with other disordered behaviours, could have resulted in a spread of more general benefits and prevention of downstream problems.

A third possibility is that participants trying to avoid aversive states may have been more aware of concrete problems in their lives and so were psychologically prepared to address their issues and change for the better. Miller \& Tonigan (1996) propose that readiness for change is based on three main factors: awareness of the problem, certainty that it is a problem, and reported changes to behaviour. They use the Stages of Change Readiness and Treatment Eagerness Scale (SOCRATES), a validated instrument used primarily for problem drinkers (Miller \& Tonigan, 1996). Using the SOCRATES to assess how prepared an individual is to change their problem drinking behaviour has been shown to be a good predictor of psychotherapy outcomes (Norcross et al., 2011). It is possible that participants who had avoid-intentions for microdosing were ready to change their lives for the better. Participants reporting avoid-intentions recognized their issues, overcame their ambivalence about their issues, and took steps (i.e. microdosing) to try to overcome their issues. The SOCRATES may be a valuable instrument to add to future microdosing research.

Ultimately, the present results should still be considered inconclusive as understanding the relations between intentions and outcomes in microdosing remains an open area for ongoing research. While the link between intentions and the benefits derived from the use of psychedelics is broadly accepted, curiously little systematic research has investigated the relationship between the two. Hartogsohn (2016) recounts the history and theory of the relationship between "set and setting" and the potential positive impact psychedelic substances can have. Specifically, Hartogsohn suggests that 
psychedelics may be "suggestibility-enhancing" and thus those who consume them expecting a positive outcome will experience better outcomes due to an enhanced placebo effect. Some empirical research suggests that in cases where higher-dose LSD was administered without care for set and setting, participants experienced worse mental health outcomes (Larsen, 2016). Similarly, a study predicting responses to psilocybin concluded that set and setting impact the subjective experience of higher doses of psilocybin: participants who were undergoing Positron Emission Tomography (PET) had significantly worse unpleasant reactions to psilocybin, ostensibly due to the uncomfortable setting (Studerus et al., 2012).

\section{The Relative Benefits of Microdoses versus Higher doses}

Respondents were split on which dose provided the most benefits: two fifths reported higher doses were somewhat or much more beneficial compared to microdosing, one third reported that microdosing was somewhat or much more beneficial compared to higher doses, one quarter reported that both were equally beneficial, and only 3-4\% of participants said they did not find either dose beneficial. Response patterns across substances were within a few percent of each other for each response option (see Figure 4). Finding that such a sizeable proportion of our sample reported that the benefits of microdosing equalled or surpassed the benefits of higher doses strongly supports continued research into microdosing.

\section{Microdosing and Anxiety Disorder}

We did not find a relationship between microdosing psychedelics and lifetime anxiety disorder diagnosis. In our previous work we found that some participants reported microdosing reduced anxiety while others reported that microdosing increased anxiety (Anderson et al., 2019). The reasons for these discrepant findings were unknown and remain unknown. We proposed that the placebo effect could be driving inconclusive results, but also that there may be individual differences in response to psychedelic microdosing including genetic or metabolic factors, personality or clinical factors, and interoceptive interpretation differences (Anderson et al., 2019). It may also be possible that different time-frames are at 
fault: lifetime diagnosis may not be related whereas more proximate or ongoing diagnoses may have more predictive power. More broadly, different responses could also be related to set and setting, as described above. For example, it may be that a safe context wherein people who microdose feel competent and supported could precipitate reduced anxiety, but in a context of normally tolerable stress or social tension, people who microdose may feel increased anxiety and uncertainty (Petranker et al., 2020). More research on the effects of microdosing in different settings is required in order to disambiguate the relationship between microdosing and anxiety.

\section{Limitations and Conclusions}

This study has several important limitations. It is a cross-sectional online survey based on respondents volunteering their time and thus may not represent the wider global population of people who microdose psychedelics. As in all self-report studies there is no way of confirming the actual substances consumed. While the sample size is quite large, surveys employ no experimental control over substance quality or dosage patterns. There could be important unmeasured effects related to dose, schedule, concurrent use of other substances, or other factors that could not be accounted for using our design. No data was collected on the patterns of microdosing which restricts exposure response analyses. Another limitation is that the survey is available in 19 languages and does not yet include large amounts of data from every country.

While this study joins a growing list of findings that suggest microdosing has a minimal risk profile and could be beneficial, the methodology employed here means that no causal claims can be made about microdosing as a practice. Future research should investigate microdosing using large sample, randomized, placebo-controlled trials over extended periods of time in order to examine the beneficial and challenging outcomes of microdosing while controlling for set and setting. 


\section{Acknowledgements}

We would like to acknowledge Norman Farb, Director of the Psychedelic Studies Research Program at the University of Toronto, who provided mentorship in the pursuit of this research. Global Drug Survey Ltd is an independent self-funded research organization. We thank the collaborating global media partners who supported GDS, country partners who translated and promoted GDS, and the study participants who volunteered their time to complete GDS. Thanks also to Chris Parsons for technical support. The National Drug and Alcohol Research Centre is supported by funding from the Australian Government under the Drug and Alcohol Program.

\section{Declaration of Conflicting Interests}

Adam R Winstock is the founder and CEO of Global Drug Survey Ltd. The other authors declare that there is no conflict of interest. 


\section{References}

Anderson, T., Petranker, R., Christopher, A., Rosenbaum, D., Weissman, C., Dinh-Williams, L.-A., Hui, K., \& Hapke, E. (2019). Psychedelic microdosing benefits and challenges: An empirical codebook. Harm Reduction Journal, 16(1), 1-10.

Anderson, T., Petranker, R., Rosenbaum, D., Weissman, C. R., Dinh-Williams, L.-A., Hui, K., Hapke, E., \& Farb, N. A. S. (2019). Microdosing psychedelics: Personality, mental health, and creativity differences in microdosers. Psychopharmacology, 236(2), 731-740. https://doi.org/10.1007/s00213-018-5106-2

Bershad, A. K., Schepers, S. T., Bremmer, M. P., Lee, R., \& de Wit, H. (2019). Acute subjective and behavioral effects of microdoses of LSD in healthy human volunteers. Biological Psychiatry. https://doi.org/10.1016/j.biopsych.2019.05.019

Carhart-Harris, R. L., \& Friston, K. J. (2019). REBUS and the Anarchic Brain: Toward a Unified Model of the Brain Action of Psychedelics. Pharmacological Reviews, 71(3), 316-344. https://doi.org/10.1124/pr.118.017160

Carhart-Harris, Robin L, Roseman, L., Haijen, E., Erritzoe, D., Watts, R., Branchi, I., \& Kaelen, M. (2018). Psychedelics and the essential importance of context. Journal of Psychopharmacology, 32(7), 725-731. https://doi.org/10.1177/0269881118754710

Davis, A. K., \& Rosenberg, H. (2017). Specific harm reduction strategies employed by 3,4methylenedioxymethamphetmine/ ecstasy users in the United States and the United Kingdom. Drug Science, Policy and Law, 3, 2050324517711069. https://doi.org/10.1177/2050324517711069

Eastwood, J. D., Frischen, A., Fenske, M. J., \& Smilek, D. (2012). The Unengaged Mind: Defining Boredom in Terms of Attention. Perspectives on Psychological Science, 7(5), 482-495. https://doi.org/10.1177/1745691612456044

Grob, C. S., Danforth, A. L., Chopra, G. S., Hagerty, M., McKay, C. R., Halberstadt, A. L., \& Greer, G. R. (2011). Pilot Study of Psilocybin Treatment for Anxiety in Patients With Advanced-Stage Cancer. Archives of General Psychiatry, 68(1), 71-78. https://doi.org/10.1001/archgenpsychiatry.2010.116

Hartogsohn, I. (2016). Set and setting, psychedelics and the placebo response: An extra-pharmacological perspective on psychopharmacology. Journal of Psychopharmacology. http://journals.sagepub.com/doi/10.1177/0269881116677852

Hutten, N. R. P. W., Mason, N. L., Dolder, P. C., \& Kuypers, K. P. C. (2019). Motives and Side-Effects of Microdosing With Psychedelics Among Users. International Journal of Neuropsychopharmacology, 22(7), 426-434. https://doi.org/10.1093/ijnp/pyz029

Johnson, M. W., Garcia-Romeu, A., Cosimano, M. P., \& Griffiths, R. R. (2014). Pilot study of the 5HT2AR agonist psilocybin in the treatment of tobacco addiction. Journal of Psychopharmacology, 28(11), 983-992. https://doi.org/10.1177/0269881114548296 
Johnson, M. W., Griffiths, R. R., Hendricks, P. S., \& Henningfield, J. E. (2018). The abuse potential of medical psilocybin according to the 8 factors of the Controlled Substances Act. Neuropharmacology. https://doi.org/10.1016/j.neuropharm.2018.05.012

Johnston, J., Barratt, M. J., Fry, C. L., Kinner, S., Stoové, M., Degenhardt, L., George, J., Jenkinson, R., Dunn, M., \& Bruno, R. (2006). A survey of regular ecstasy users' knowledge and practices around determining pill content and purity: Implications for policy and practice. International Journal of Drug Policy, 17(6), 464-472. https://doi.org/10.1016/j.drugpo.2006.03.008

Larsen, J. K. (2016). Neurotoxicity and LSD treatment: A follow-up study of 151 patients in Denmark. History of Psychiatry, 27(2), 172-189. https://doi.org/10.1177/0957154X16629902

Miller, W. R., \& Tonigan, J. S. (1996). Assessing drinkers' motivation for change: The Stages of Change Readiness and Treatment Eagerness Scale (SOCRATES). Psychology of Addictive Behaviors, 10(2), 81-89. http://dx.doi.org.myaccess.library.utoronto.ca/10.1037/0893-164X.10.2.81

Norcross, J. C., Krebs, P. M., \& Prochaska, J. O. (2011). Stages of change. Journal of Clinical Psychology, 67(2), 143-154. https://doi.org/10.1002/jclp.20758

Nutt, D. J., King, L. A., \& Phillips, L. D. (2010). Drug harms in the UK: A multicriteria decision analysis. The Lancet, 376(9752), 1558-1565. https://doi.org/10.1016/S0140-6736(10)61462-6

Petranker, R., Kim, J., \& Anderson, T. (2020). Microdosing as a response to the meaning crisis. https://doi.org/10.31234/osf.io/2jnkf

Plante, S. G. (2017, April 24). LSD microdoses make people feel sharper, and scientists want to know how. The Verge. https://www.theverge.com/2017/4/24/15403644/microdosing-lsd-acidproductivity-benefits-brain-studies

Polito, V., \& Stevenson, R. J. (2019). A systematic study of microdosing psychedelics. PLOS ONE, 14(2), e0211023. https://doi.org/10.1371/journal.pone.0211023

Pollan, M. (2018). How to Change Your Mind: What the New Science of Psychedelics Teaches Us About Consciousness, Dying, Addiction, Depression, and Transcendence. Penguin.

Prochazkova, L., Lippelt, D. P., Colzato, L. S., Kuchar, M., Sjoerds, Z., \& Hommel, B. (2018). Exploring the effect of microdosing psychedelics on creativity in an open-label natural setting. Psychopharmacology, 235(12), 3401-3413. https://doi.org/10.1007/s00213-018-5049-7

Ross, S., Bossis, A., Guss, J., Agin-Liebes, G., Malone, T., Cohen, B., Mennenga, S. E., Belser, A., Kalliontzi, K., Babb, J., Su, Z., Corby, P., \& Schmidt, B. L. (2016). Rapid and sustained symptom reduction following psilocybin treatment for anxiety and depression in patients with life-threatening cancer: A randomized controlled trial. Journal of Psychopharmacology, 30(12), 1165-1180. https://doi.org/10.1177/0269881116675512

Studerus, E., Gamma, A., Kometer, M., \& Vollenweider, F. X. (2012). Prediction of psilocybin response in healthy volunteers. PloS One, 7(2), e30800. https://doi.org/10.1371/journal.pone.0030800

Suzuki, J., Dekker, M. A., Valenti, E. S., Arbelo Cruz, F. A., Correa, A. M., Poklis, J. L., \& Poklis, A. (2015). Toxicities Associated With NBOMe Ingestion-A Novel Class of Potent Hallucinogens: A Review of the Literature. Psychosomatics, 56(2), 129-139.

https://doi.org/10.1016/j.psym.2014.11.002 
Waldman, A. (2017). A Really Good Day: How Microdosing Made a Mega Difference in My Mood, My Marriage, and My Life. Knopf.

Weiss, H. (2019, March 5). A New Chapter in the Science of Psychedelic Microdosing. The Atlantic. https://www.theatlantic.com/health/archive/2019/03/psychedelic-microdosing-depressionanxiety/584119/ 